\title{
Electroacupuncture and Transcutaneous Electrical Nerve Stimulation in Chronic Nonspecific Low Back Pain: a Blind Randomized Clinical Trial
}

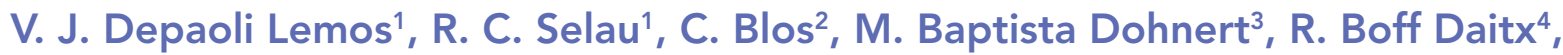 \\ V. de Almeida Brito ${ }^{4}$ \\ 1 Physiotherapy Course, Lutheran University of Brazil, Torres, Brazil \\ 2 Physiotherapist, Municipal Public Servant, Arroio do Sal, Brazil \\ 3 Research, Teaching and Extension Laboratory in Orthopedic Trauma Physiotherapy (LAPEFITO), Gurupi \\ University (UnirG), Gurupi, Torres, Brazil \\ 4 Lutheran University of Brazil (Ulbra), Brazil
}

\section{CORRESPONDING AUTHOR:}

Vicente de Almeida Brito

Lutheran University of Brazil (Ulbra)

Rua Manoel Fortunato de Souza 694

Bairro Getúlio Vargas, Torres (RS), Brasil

E-mail: vicentebrito09@gmail.com

DOI:

10.32098/mltj.04.2021.15

LEVEL OF EVIDENCE: $1 B$

REBEC REGISTRATION:

RBR-9w54gd

\begin{abstract}
SUMMARY
Background. Chronic nonspecific low back pain impairs function in affected individuals. Transcutaneous Electrical Nerve Stimulation (TENS) has shown to be effective in reducing the intensity of chronic nonspecific low back pain and should be used as a complementary treatment. The same can be said for electroacupuncture (EA), which consists of the application of electrical stimulation through punctured needles in acupuncture meridians, generating physiological reactions and leading to therapeutic effects.

Objective. To compare the effects of EA and TENS in subjects with chronic nonspecific low back pain.

Methods. Blind randomized clinical trial of 48 subjects with chronic nonspecific low back pain. The patients were allocated to the following groups: Conventional Kinesiotherapy (CG), Conventional Kinesiotherapy plus Transcutaneous Electrical Nerve Stimulation (CTENSG), and Conventional Kinesiotherapy plus Electroacupuncture (CEAG). The individuals were evaluated before and after interventions and at a 30-day follow-up for the following factors: pain level, flexibility, lumbopelvic stability, and function. A total of ten interventions were performed three times a week for four weeks.

Results. All groups significantly improved pain. However, CEAG reduced pain significantly more than GTENSG and CG $(\mathrm{p}<0.05)$. The three groups significantly improved function at endpoint $(\mathrm{p}<0.05)$. Regarding the Roland Morris questionnaire, GCEAC scored significantly lower than CTENSG and CG $(\mathrm{p}<0.05)$. Lumbopelvic stability improved in all tests for CEAG and CG.

Conclusions. The association between electroacupuncture and exercise improved pain, function, and lumbopelvic stability in comparison to exercise alone or in association with TENS.
\end{abstract}

KEY WORDS

Low Back Pain; electroacupuncture; Transcutaneous Electrical Nerve Stimulation; physiotherapy; Exercise Therapy.

\section{INTRODUCTION}

Low back pain is a public health problem worldwide. Its prevalence rate is $80 \%$ (1), being one of the most common and disabling musculoskeletal disorders in the world $(1,2)$. In some patients, initial acute pain can last for three months and eventually develop into chronic low back pain (1). The diagnosis is usually defined as pain below the costal margin and above the inferior 
gluteal folds, characterizing specific or nonspecific low back pain (3).

Nonspecific low back pain is defined as a symptom for which there is currently no reliable method to identify the pathology. It affects approximately $70 \%$ to $85 \%$ of the population at some stage in their lives (4). Psychosocial factors play a role in the development and maintenance of this condition (5), where a wide variety of factors have some influence. It leads to functional disability, limited general mobility, worse self-reported health, lower quality of life, absenteeism in the workplace, and depression $(5,6)$. Low back pain patients often report limitations in their daily activities, which affects interpersonal relationships and socialization that are important for any individual (5).

Etiology can be subdivided into mechanical, systemic, and referred groups. The most frequent cause is mechanic (97\%), generating "nonspecific low back pain" (4). This definition is used when the cause of pain cannot be precisely determined, thus excluding those cases of patients presenting with a specific cause (e.g., fracture, infection, cancer) (4).

Physiotherapy aims to improve the functional capacity and quality of life of these patients (7), and exercise is an effective alternative to reduce pain (8). Transcutaneous electrical nerve stimulation (TENS) is also effective in reducing the intensity of nonspecific low back pain and should be used as a complementary treatment (9). Among its modalities of application is the acupuncture mode (TENS-AC), which uses an electrical stimulation with low frequency $(<10 \mathrm{~Hz})$, high pulse width (> $150 \mathrm{mp}$ ), and high intensity, respecting the patient's tolerance. This mode (TENS-AC) stimulates $\mathrm{A} \delta$ and $\mathrm{C}$ nociceptive fibers, decreasing pain from the activation of endogenous opioid mechanisms known as diffuse noxious inhibitory controls (9).

Another method used is electroacupuncture (EA), which consists of the application of electrical stimulation through puncture needles in acupuncture meridians, generating physiological reactions and leading to therapeutic effects such as analgesia (10). Electroacupuncture (EA) modulates pain through significant changes in opioids, serotonin, and norepinephrine, which are stimulated to defined sites, acting in the spinal cord and supraspinal structures (11). Electroacupuncture (EA) derived from the integration of traditional acupuncture and modern electrical stimulation is generally accepted because it is a relatively direct, safe, and inexpensive therapy in comparison to other conventional therapies (12). In addition, EA has become increasingly used in clinical practice due to its repeatability and standardization of frequency, intensity, and duration. After the needles are inserted into the acupuncture points, the electrodes are connected to the pairs of needles and then a small electrical current is administered (12).

Considering that nonspecific low back pain is one of the main causes of disabilities today, the present study compared the effects of EA and TENS in subjects affected by this condition.

\section{METHODS}

Blind randomized clinical trial of patients with chronic nonspecific low back pain. Subjects of both sexes and aged between 18 and 70 years were recruited. Sample calculation considered the immediate effect of TENS on pain reduction in patients with chronic nonspecific low back pain as the primary outcome of the study. Following Ebadi et al. (13), for an initial sample calculation, we used a study power of $80 \%$, a significance level of $95 \%$, a sampling error of $5 \%$, and a sample size ratio of 1: 1: 1 (Kinesiotherapy group - CG: Kinesiotherapy and TENS group - CTENSG: Kinesiotherapy and Electroacupuncture group - CEAG), reaching the estimated number of 14 subjects for each intervention group. Believing that losses and refusals would be around $15 \%$, we reached the final number of 16 subjects for each study group, totaling 48 subjects.

Inclusion criteria were as follows: medical prescription for physiotherapeutic treatment of nonspecific low back pain, history of low back pain for more than three months, and no other type of concurrent treatment. Subjects with three absences, with history of previous surgery on the lumbar spine, or with imaging exams showing intervertebral degenerative process and pain radiated to the lower limbs were excluded. This research was approved by the Ethics and Research Committee of the Lutheran University of Brazil under opinion No. 3,738,209. All participants previously signed an Informed Consent Form (ICF). The study was registered in the Brazilian Registry of Clinical Trials (REBEC) under the number RBR-9w54gd. This study meets the ethical standards of Muscles, Ligaments and Tendons Journal (14).

\section{Data collection, evaluation and randomization}

After fulfilling the eligibility criteria, the study participants received information about the research and were evaluated by a previously trained evaluator who did not know to which group the subject had been assigned (blind evaluator). Evaluations took place before and after intervention and thirty days after the intervention.

An evaluation form was used to collect sociodemographic data. The visual analog scale (VAS) was used to assess pain level. The Wells Bench was used to assess posterior chain flexibility, as described by Pitanga (15). Lumbopelvic stability was assessed using the static trunk endurance test, in which the subject seats with hips and knees flexed at $90^{\circ}$, arms folded and crossed at the chest, and feet fixed by the evaluator, with the trunk at an angle of $60^{\circ}$. In this test, the subject holds the isometric posture for as long as possible and the examiner records the posture maintenance time. The Sorensen endurance test and the side bridge test were also performed. 
Function was assessed using the Roland-Morris Disability Questionnaire (RMDQ). This questionnaire consists of 24 self-answer questions, which participants complete in less than five minutes. The questions have a dichotomous answer (yes or no) and the final result corresponds to the sum of the yes answers. This result can vary between 0 and 24 , with zero corresponding to a person without complaints and the maximum value corresponding to a patient with very severe limitations. After going through the initial assessment, the subjects were assigned by an independent collaborating researcher, that is, who did not participate in the other phases of the study. Randomization took place using sealed envelopes corresponding to each of the groups to which the subject was assigned: Kinesiotherapy group (CG), Kinesiotherapy and TENS group (CTENSG), and Kinesiotherapy and Electroacupuncture group (CEAG).

\section{Intervention program}

Three interventions were performed weekly for four weeks, totaling ten interventions. Consultations lasted 50 minutes, with vital signs being checked before and after intervention. Heart rate $(\mathrm{HR})$ and peripheral $\mathrm{O}_{2}$ saturation were measured using a G-Tech ${ }^{\circledR}$ LED oximeter. Blood pressure was measured using a G-Tech ${ }^{\circledR}$ LED sphygmomanometer and a Littman ${ }^{\circledR}$ stethoscope, both previously calibrated. Finally, respiratory rate was measured using a Western ${ }^{\circledR}$ digital chronometer. Participants from the conventional kinesiotherapy group (CG) initially performed three 20 -second sets of stretches for posterior muscle chain, followed by 10 repetitions of strengthening exercises for core stabilizing muscles (held for 6" in the first and second weeks, 8 " in the third week, and 10 " in the fourth week). These exercises were: supine bridge, single leg supine bridge, side bridge, prone plank (table I).

Participants in the kinesiotherapy and TENS group (CTENSG) underwent the same kinesiotherapy program but with TENS application after kinesiotherapy. A portable two-channel transcutaneous electrostimulator (brand Ibramed ${ }^{\circledR}$, model Neurodyn; ANVISA registration number 10360310012) was used. Before applying the electrodes, the skin of the participants was cleaned using cotton and 70\% alcohol gel. Total application time was 20 minutes. The parameters were pulse width of 250 $\mu \mathrm{s}$, pulse frequency of $10 \mathrm{~Hz}$, and current intensity according to the participant's tolerance. Two channels with four $5 \times 5 \mathrm{~cm}$ self-adhesive Valutrode electrodes were used, positioned bilaterally in the paravertebral musculature (L1 and $\mathrm{L} 5$ region).

In the CEAG, participants underwent the same kinesiotherapy program as the previous groups, but with the application of electroacupuncture after kinesiotherapy. For that, an acupuncture electrostimulator (brand Sikuro ${ }^{\circledR}$, model DS100jr; ANVI-

Table I. Description of the kinesiotherapy program.

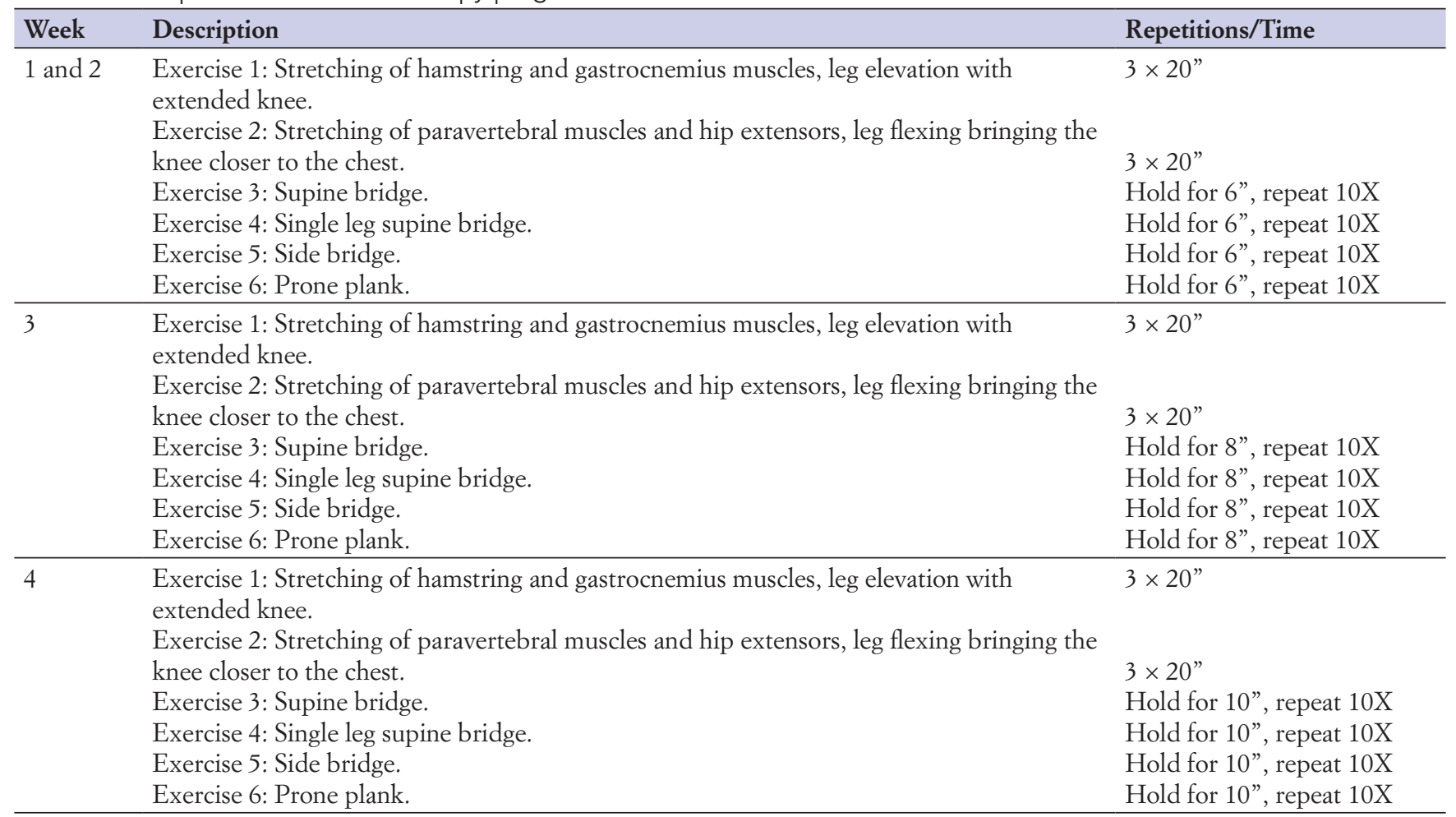


SA MS registration number 80470920001) was used. Before application, the puncture area was cleaned with cotton and $70 \%$ alcohol gel. Then, $25 \times 40 \mathrm{~mm}$ needles (brand Gold Dragon $\left.{ }^{\circledR}\right)$ were used for puncture at bladder meridian points B22 (L1) and B26 (L5). The same reference points of the intervention in CTENSG were maintained. Total procedure time was 20 minutes. For this procedure, signal 1 was used: continuous pulse train, with pulse frequency of $10 \mathrm{~Hz}$. Current intensity was administered according to the participant's tolerance, using E-JS18 cables ('alligator'-type electrodes) to connect the device to the puncture needles. Two channels were used with four stimulator cables connected to the needles. After intervention, the needles were immediately discarded in a Descarpack box duly appropriated for this purpose.

\section{Statistical analysis}

The Statistical Package for the Social Sciences (SPSS) version 23.0 was used for data analysis. Initially, a descriptive analysis of the study variables was performed through absolute value, frequency, mean, and standard deviation. Afterwards, the variables were statistically analyzed by parametric tests. Analysis of Variance (ANOVA) for repeated measures was used within each group from baseline to follow-up assessments, and unpaired Student $t$ test for analysis of the variables between groups at each moment. For nonparametric variables, the Kruskal-Wallis and Mann-Whitney tests were used, respectively. The level of significance established for the statistical test was $\mathrm{p}<0.05$.

\section{RESULTS}

The initial evaluation included 54 subjects. Of these, 6 were excluded for not meeting the eligibility criteria. Therefore, 48 subjects were randomized, 16 for each group, as shown in figure 1.

Table II shows the baseline characteristics of the participants in the study groups. The groups were homogeneous for the analyzed variables (table II).

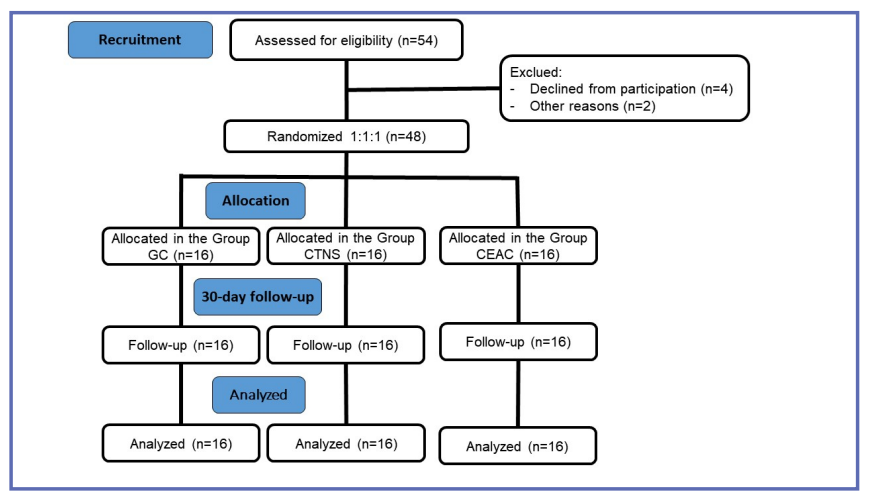

Table II. Characterization of study subjects $(n=48)$.

\begin{tabular}{|c|c|c|c|c|}
\hline \multicolumn{5}{|c|}{ Intervention Group } \\
\hline & $\begin{array}{l}\text { CTENSG } \\
(\mathrm{n}=16)\end{array}$ & $\begin{array}{l}\text { CEAG } \\
(n=16)\end{array}$ & $\begin{array}{l}\text { CG } \\
(n=16)\end{array}$ & p value \\
\hline Age, years $(m \pm s d)$ & $52.50 \pm 12.42$ & $45.37 \pm 13.51$ & $50.81 \pm 12.96$ & $0.28 *$ \\
\hline Gender, n (\%) & & & & $0.49 * *$ \\
\hline Male & $3(18.75)$ & $6(37.5)$ & $5(31.25)$ & \\
\hline Female & $13(81.25)$ & $10(62.5)$ & $11(68.75)$ & \\
\hline Skin color, n (\%) & & & & $0.76 * *$ \\
\hline White & $15(93.75)$ & $14(87.5)$ & $15(93.75)$ & \\
\hline Black & $1(6.25)$ & $2(12.5)$ & $1(6.25)$ & \\
\hline Weight, kg $(\mathrm{m} \pm \mathrm{sd})$ & $76.62 \pm 11.57$ & $76.44 \pm 10.49$ & $74.19 \pm 10.70$ & $0.84 *$ \\
\hline Height, $\mathrm{m}(\mathrm{m} \pm \mathrm{sd})$ & $1.62 \pm 0.09$ & $1.67 \pm 0.09$ & $1.65 \pm 0.07$ & $0.18^{*}$ \\
\hline BMI, $n(m \pm s d)$ & $28.95 \pm 4.32$ & $27.36 \pm 4.00$ & $27.29 \pm 3.11$ & $0.39 *$ \\
\hline Occupation, n (\%) & & & & $0.23 * *$ \\
\hline Housekeeper & $6(37.6)$ & $3(18.8)$ & $5(31.3)$ & \\
\hline General services & $6(37.6)$ & $10(62.1)$ & $5(31.3)$ & \\
\hline Retired & $3(18.8)$ & $0(0.0)$ & $2(12.4)$ & \\
\hline Other & $1(6.0)$ & $3(18.8)$ & $4(25.0)$ & \\
\hline Time of pain, years $(\mathrm{m} \pm \mathrm{sd})$ & $5.56 \pm 3.72$ & $7.37 \pm 5.47$ & $5.00 \pm 3.59$ & $0.28^{*}$ \\
\hline
\end{tabular}

Kg: kilogram; m: meter; " One-way ANOVA; **Chi-Square. 
All intervention groups improved pain (VAS) from baseline to follow-up. The CEAG group had a significantly lower pain score than groups CTENSG and CG both in the final assessment and in the follow-up assessment $(\mathrm{p}<0.05)$.

Posterior chain flexibility, assessed through the Wells Bench, improved significantly from baseline to endpoint only in CG $(\mathrm{p}<0.01)$. When comparing groups, CEAG and CG had a higher level of flexibility than CTENSG in the final and follow-up assessments ( $\mathrm{p}<0.05)$ (table III).

Lumbopelvic stability tests showed an increase in posture maintenance in CEAG and CG from baseline to endpoint. The CTENSG group improved scores only in the static trunk endurance test. The comparison between groups showed that CEAG subjects maintained the correct posture significantly longer than CTENSG and CG subjects in the follow-up assessment (table IV).

Function assessment using the RMDQ showed that the three groups significantly improved scores from baseline to endpoint and follow-up. However, CEAG showed a significantly lower score than CTENSG and CG in the final and follow-up assessments $(\mathrm{p}<0.05)($ table $\mathbf{V})$.

\section{DISCUSSION}

The present study compared the effects of EA and TENS on pain in subjects with nonspecific low back pain. Recent studies on the same pathology showed sample homogeneity (16-18). That said, the subjects of this study were homogeneous for gender, age, skin color, Body Mass Index (BMI), occupation, and time of pain.
Our research followed a kinesiotherapy protocol similar to the method described by Mendes et al. (19), who also obtained positive results in improving pain and function in the kinesiotherapy group. For the CTENSG intervention, the TENS parameters applied were similar to those of the study by Tousingnat-Laflamme et al. (9), in which pain also improved significantly. For the CEAG intervention, the applied electroacupuncture parameters were similar to those of the study by Comachio (16). In that study, pain improved significantly with the use of electroacupuncture and manual acupuncture as a complementary treatment for low back pain. These results corroborate those obtained for CEAG, with pain improvement after intervention and significant results in pain relief in three months of followup. Electroacupuncture (EA) modulates pain through significant changes in opioids, serotonin, and norepinephrine, which are inhibitory neurotransmitters in the dorsal horn of the spinal cord, activated by electrical stimuli at the acupuncture points to act in the spinal cord and supraspinal structures (11).

When comparing intergroup results, pain improved significantly in CEAG in comparison to CTENSG and CG in the final and follow-up assessments. Likewise, Leite et al. (17) conducted a randomized clinical trial to verify whether electroacupuncture is effective in reducing pain and in quantitative responses to sensory tests in patients with chronic nonspecific low back pain. The authors used an electroacupuncture treatment group and three different control groups, with a total of 69 participants. As a conclusion, the electroacupuncture group significantly reduced

Table III. Pain score (VAS) and posterior chain flexibility in the study groups.

\begin{tabular}{|c|c|c|c|c|}
\hline & \multicolumn{3}{|c|}{ Intervention Group } & \multirow[b]{2}{*}{ p value } \\
\hline & $\begin{array}{c}\text { CTENSG } \\
(\mathrm{n}=16)\end{array}$ & $\begin{array}{l}\text { CEAG } \\
(n=16)\end{array}$ & $\begin{array}{c}C G \\
(n=16)\end{array}$ & \\
\hline Baseline & $8.88 \pm 1.03$ & $8.75 \pm 0.93$ & $9.19 \pm 0.83$ & 0.40 \\
\hline Endpoint & $3.56 \pm 2.85$ & $1.25 \pm 1.18$ & $3.31 \pm 2.85$ & $0.02^{\# \$}$ \\
\hline \multicolumn{5}{|c|}{ Posterior chain flexibility, cm } \\
\hline Baseline & $14.44 \pm 4.98$ & $19.88 \pm 8.23$ & $20.38 \pm 8.62$ & 0.05 \\
\hline Endpoint & $17.13 \pm 7.35$ & $24.13 \pm 8.55$ & $24.69 \pm 7.10$ & $0.01^{\# \& \alpha}$ \\
\hline Follow-up & $16.06 \pm 7.51$ & $24.00 \pm 9.83$ & $24.25 \pm 6.57$ & $0.009^{\# 8}$ \\
\hline
\end{tabular}

$\mathrm{Cm}$ : centimeters; ${ }^{\mathrm{a}} \mathrm{p}<0.01$ when comparing baseline with endpoint. ANOVA for repeated measures; ${ }^{\mathrm{b}} \mathrm{p}<0.01$ when comparing baseline with follow-up. ANOVA for repeated measures; ${ }^{p} \mathrm{p}<0.05$ between CTENSG and CEAG. One-Way ANOVA; ${ }^{\$} \mathrm{p}<0.05$ between CEAG and CG. One-Way ANOVA; ${ }^{*} \mathrm{p}$ $<0.05$ between CTENSG and CG. One-Way ANOVA. 
Table IV. Results of lumbopelvic stability tests in the study groups.

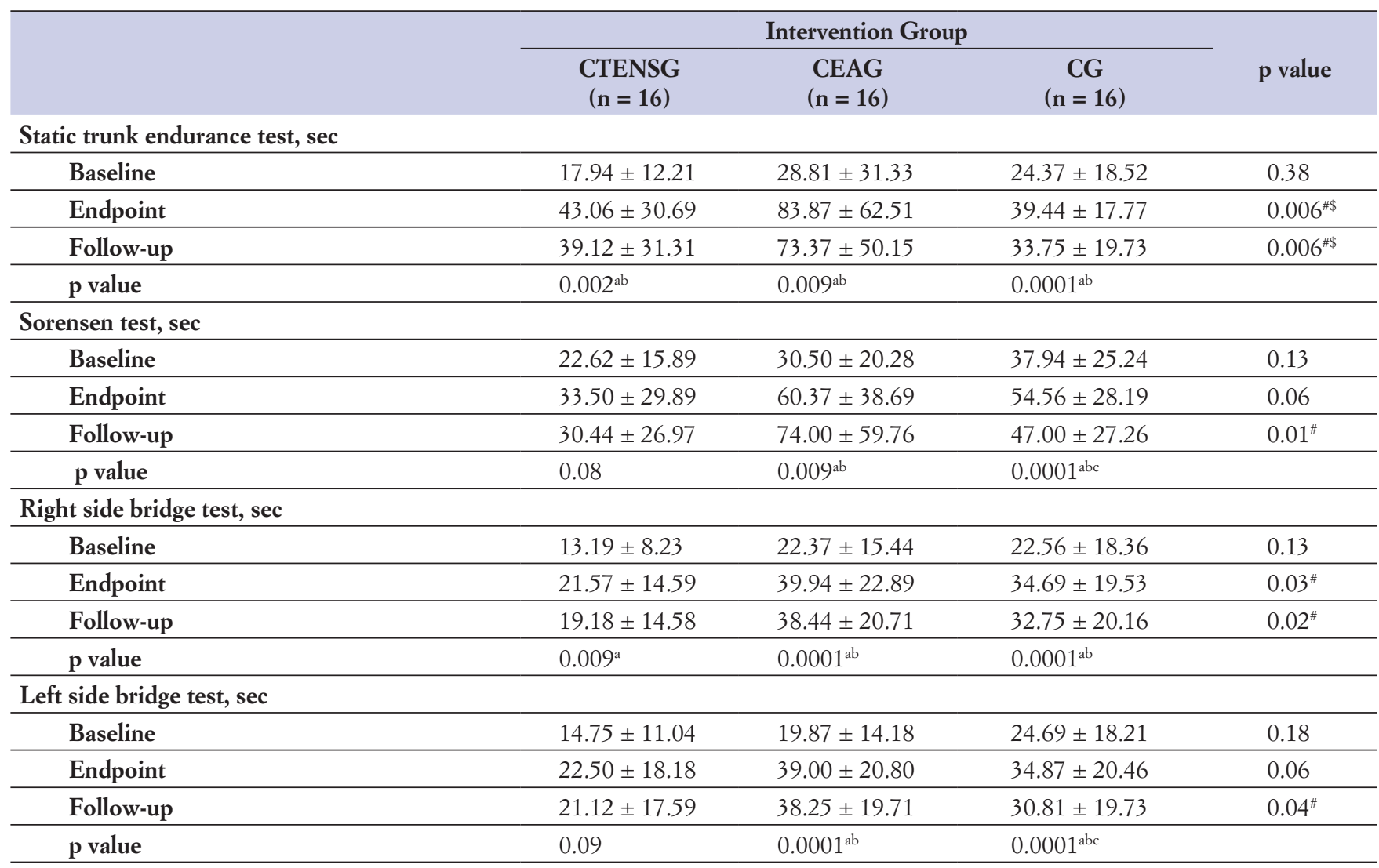

Sec: seconds; ${ }^{\mathrm{a}} \mathrm{p}<0.05$ when comparing baseline with endpoint. ANOVA for repeated measures; ${ }^{\mathrm{b}} \mathrm{p}<0.05$ when comparing baseline with follow-up. ANOVA for repeated measures; ${ }^{c} \mathrm{p}<0.05$ when comparing endpoint with follow-up. ANOVA for repeated measures; ${ }^{*} \mathrm{p}<0.05$ between CTENSG and CEAG. One-Way ANOVA; ${ }^{\$} \mathrm{p}<0.05$ between CEAG and CG. One-Way ANOVA; ${ }^{\circledR} \mathrm{p}<0.05$ between CTENSG and CG. One-Way ANOVA.

Table V. RMDQ scores in the study groups.

\begin{tabular}{|c|c|c|c|c|}
\hline & \multicolumn{3}{|c|}{ Intervention Group } & \multirow[b]{2}{*}{ p value } \\
\hline & $\begin{array}{c}\text { CTENSG } \\
(\mathrm{n}=16)\end{array}$ & $\begin{array}{c}\text { CEAG } \\
(n=16)\end{array}$ & $\begin{array}{c}C G \\
(n=16)\end{array}$ & \\
\hline Baseline & $16.63 \pm 3.96$ & $16.50 \pm 4.41$ & $19.81 \pm 3.23$ & $0.03^{\$}$ \\
\hline Endpoint & $10.31 \pm 7.13$ & $4.44 \pm 5.67$ & $9.88 \pm 5.62$ & $0.02^{\# \&}$ \\
\hline
\end{tabular}

${ }^{\mathrm{a}} \mathrm{p}=0.0001$ when comparing baseline with endpoint. ANOVA for repeated measures; ${ }^{\mathrm{b}} \mathrm{p}=0.0001$ when comparing baseline with follow-up. ANOVA for repeated measures; ${ }^{c} p<0.05$ when comparing endpoint with follow-up. ANOVA for repeated measures; ${ }^{*} p<0.05$ between CTENSG and CEAG. One-Way ANOVA; ${ }^{\$} \mathrm{p}<0.05$ between CEAG and CG. One-Way ANOVA; ${ }^{\star} \mathrm{p}<0.05$ between CTENSG and CG. One-Way ANOVA.

pain at rest and on exertion. Dohnert et al. (20) described pain reduction after four weeks of lumbopelvic stabilization and Mckenzie exercises. The authors state that the analgesic effects generated by different types of exercises can have different explanations (20). For example, the application of controlled forces to the spine through exercise can temporarily reduce pain intensity, altering the fluid dynamics of the injured tissue (20). Another justification would be that lumbopelvic stabilization exercises can reduce the load and improve the quality of movements after improving the coor- 
dination of trunk muscles (20). In addition, stabilization exercises mainly activate deep muscles, commonly affected by low back pain (20). Choopani et al. (21) treated 24 patients with low back pain secondary to spondylolisthesis twice a week for two months. The patients were divided into a group of conventional exercises and a group of lumbopelvic stabilization exercises. The authors observed that both programs reduced pain and disability, with no differences between groups (21).

Corroborating the analgesic effect of electroacupuncture in this research, Awad et al. (22) compared the effect of EA and low-level laser therapy on postpartum low back pain. The study included 50 women with complaints of low back pain, divided into a group with 25 women treated with electroacupuncture three times a week and a group with 25 women treated with low level laser therapy three times a week. The visual analog scale was used to measure pain intensity before and after treatment. The authors concluded that electroacupuncture is more effective in reducing pain than low level laser therapy.

Function improved significantly in the three groups from baseline to endpoint and follow-up. This corroborates the findings of Comachio et al. (16), who carried out a study to evaluate the efficacy of electroacupuncture and manual acupuncture on pain and function in patients with low back pain. These authors concluded that both treatments have similar effects, reducing pain and improving function in participants.

Therefore, electroacupuncture is effective in improving function. Following this line of thought, CEAG had a significantly better score in function in comparison to CTENSG and CG in the final and follow-up assessments. These findings corroborate with Kong et al. (23), who evaluated the effect of electroacupuncture and placebo electroacupuncture on pain and function in adults with low back pain. The authors found a significant improvement in function in the electroacupuncture group.

Electroacupuncture promotes positive regulation of adenosine and increases the inhibitory effects induced by adenosine in substance $\mathrm{P}$, generating clinical benefits (24). The technique also improves sensory symptoms and regulates pain through neurophysiological mechanisms that activate sympathetic nervous fibers to increase endogenous opioids at the site of pain (25).

Activation of sympathetic nervous fibers increases the expression of the intracellular adhesion molecule in the blood vessels of inflamed tissue to promote the migration of polymorphonuclear leukocytes containing $\beta$-endorphin, metenkephalin, and mononuclear cells. Moreover, the activation of sympathetic neuron-derived norepinephrine stimulates adrenergic receptors in inflammatory cells to release $\beta$-endorphin, reducing pain (25) and improving function.

Only CG significantly improved posterior chain flexibility. In the comparison between groups, CEAG and CG showed higher flexibility than CTENSG in the final and follow-up assessments. Corroborating with the results of the present research, the study by Dohnert et al. (26) assessed 30 female participants with chronic low back pain. Study participants were divided into three groups: CORE exercises (CG), neuromuscular electrical stimulation (NMES) (NG), and CORE exercises in association with NMES (CNG). The authors obtained a significant increase in flexibility after intervention in the CORE group and in the CORE + NMES group, but only the CORE group maintained the level of flexibility in the follow-up assessment (26).

Song et al. (27) investigated the effect of TENS in association with static stretching on hamstring flexibility. One group of participants received three sessions of static stretching interventions, while the other group performed static stretching in association with TENS. All groups significantly improved hamstring flexibility. The study thus demonstrates that kinesiotherapy significantly improves flexibility and that adding electroacupuncture to the protocol can enhance this improvement in individuals with nonspecific low back pain. Core stability exercises reduce the intravertebral load and improve the quality of movements by improving trunk muscle coordination, with positive effects on flexibility (25). These exercises mainly activate deep muscles, commonly affected by pain in the lumbar region. Electroacupuncture generates analgesic effects in these muscles so that they can be more quickly activated to carry out their biomechanical functions.

The results of lumbopelvic stability tests showed an increase in posture maintenance in CEAG and CG from baseline to endpoint. In the comparison between groups, CEAG participants maintained the correct posture for longer than CTENSG and CG participants in the follow-up assessment. This finding agrees with Armando et al. (28) in their study that compared TENS and stabilization exercises to prevent fatigue and improve muscle activation in patients with low back pain due to herniated disc. The authors concluded that although TENS relieves pain, it is not effective as a single therapy since stabilization exercises alone improved all the measured results. Regardless of the support of electrophysical agents, core stability exercises for patients with low back pain improve the size and recruitment of deep spinal muscles such as $\operatorname{Tr} A$, improving pain and function in the short term (20).

Dohnert et al. (26) also investigated lumbopelvic stability tests in a randomized, double-blind clinical trial in which intervention groups were divided into kinesiotherapy with 
CORE exercises and kinesiotherapy with CORE exercises + NMES. Both groups improved posture maintenance time in all tests in the final and follow-up assessments. Kinesiotherapy exercises are thus effective in improving lumbopelvic stability, regardless of associated electrotherapy.

The proposed mechanism of action for core stability exercises improves motor control and the motor coordination of deep muscles of the spine and trunk such as the transversus abdominis, internal oblique, and rectus abdominis (28). Activation of these muscles improves stability in the points of origin of the segmental muscles and positively affects lumbopelvic stability (29). However, doubts still exist regarding the results of the association of therapeutic electrical currents in the approach for low back pain. Results are still controversial due to variations in stimulation parameters, demographic and anthropometric characteristics of participants, study design, outcome measures, and duration and planning of interventions. Laybidy et al. (30) are conducting a systematic review to clarify which electrical stimulation current improves pain and function in chronic nonspecific low back pain. The results can be valuable in clinical practice to optimize therapeutic planning.

\section{Limitations}

Our study has some limitations that may limit the extrapolation of results. Initially, the sample (despite having been calculated) is small. In addition, the short follow-up prevents us from analyzing these results in the long term.

\section{REFERENCES}

1. Suh JH, Kim H, Jung GP, et al. The effect of lumbar stabilization and walking exercises on chronic low back pain: A randomized controlled trial. Medicine (Baltimore) 2019;98(26):16173.

2. Grabovac I, Dorner TE. Association between low back pain and various everyday performances. Wien Klin Wochenschr 2019;131(21-22):541-9.

3. Tatsunori I, Kenji M, Takako M, et al. Psychological Treatment Strategy for Chronic Low Back Pain. Spine Surg Relat Res 2018;10(3):199-206.

4. Gianola S, Castellini G, Andreano A, et al. Effectiveness of treatments for acute and sub-acute mechanical non-specific low back pain: protocol for a systematic review and network meta-analysis. Systematic Reviews 2019;8(1):196.

5. Barbosa FM, Vieira ÉBM, Garcia JBS. Beliefs and attitudes in patients with chronic low back pain. Br J Pain 2018;1(2):116-21.

6. Cichoń D, Ignasiak Z, Fugiel J, et al. Efficacy of Physiotherapy in Reducing Back Pain and Improve Joint Mobility in Older Women. Ortop Traumatol Rehabil 2019; 21(1):45-55.

7. Arins MR, Murara N, Bottamedi X. Physiotherapeutic treatment Schedule for chronic low back pain: influence
We interpret that, although CEAG had presented better results in the evaluated items, kinesiotherapy may have influenced the results of CTENSG. Even considering that the results demonstrate the effectiveness of each of these techniques, we understand that new studies adding a control group and groups contemplating more parameters of regulation of electrical currents must be carried out to develop better guidelines for the treatment of nonspecific low back pain. Finally, we showed few records of recent studies using EA in low back pain, which makes it difficult to have a wide-ranging discussion about the technique.

\section{CONCLUSIONS}

Exercises proved to be the basis for the treatment of chronic nonspecific low back pain. The association between EA and exercise significantly improved pain, function, and lumbopelvic stability in comparison to exercise alone or in association with TENS. Based on the results of this clinical trial, EA can be used as an adjunct to lumbopelvic stability exercises in the clinical practice of low back pain. This would provide patients with a more effective reduction of symptoms and consequent enhancement of the proposed exercise.

\section{CONFLICT OF INTERESTS}

The authors declare that they have no conflict of interests.

on pain, quality of life and functional capacity. Rev Dor 2016;17(3):192-6

8. Filho JNS, Gurgel JL, Porto F. Influence of stretching exercises in musculoskeletal pain in nursing professionals. Fisioter Mov 2020;33:e003317.

9. Laflamme YT, Laroche C, Beaulieu C, et al. A randomized trial to determine the duration of analgesia following a 15 and a 30-minute application of acupuncture-like TENS on patients with chronic low back pain. Physiother Theory Pract 2017;33:361-9.

10. Toroski M, Nikfar S, Mojahedian MM, et al. Comparison of the Cost-utility Analysis of Electroacupuncture and Nonsteroidal Antiinflammatory Drugs in the Treatment of Chronic Low Back Pain. J Acupunct Meridian Stud 2018;11(2):62-6.

11. Shin BC, Cho JH, Ha IH, et al. A multi-center, randomized controlled clinical trial, cost-effectiveness and qualitative research of electroacupuncture with usual care for patients with non-acute pain after back surgery: study protocol for a randomized controlled trial. Trials 2018;19(1):65.

12. Zhan J, Pan R, Zhou M, et al. Electroacupuncture as an adjunctive therapy for motor dysfunction in acute stroke 
survivors: a systematic review and meta-analyses. BMJ Open 2018;8(1):e017153.

13. Ebadi S, Ansari, NN, Ahadi T, Fallah E, Forogh B. No immediate analgesic effect of diadynamic current in patients with nonspecific low back pain in comparison to TENS. J Bodyw Mov Ther 2018;22(3):693-9.

14. Padulo J, Oliva F, Frizziero A, Maffuli N. Basic principles and recommendations in clinical and field Science Research: 2018 update. Muscles Ligaments Tendons J 2018;8(3):305-7.

15. Pitanga FJG. Teste, Medidas e Avaliação em Educação Física e Esportes. 5. ed. São Paulo: Phorte 2008.

16. Comachio J, Oliveira CC, Silva IFR, et al. Effectiveness of Manual and Electrical Acupuncture for Chronic Non-specific Low Back Pain: A Randomized Controlled Trial. J Acupunct Meridian Stud 2020;13(3):87-93.

17. Leite PMS, Mendonça ARC, Maciel LYS, et al. Does Electroacupuncture Treatment Reduce Pain and Change Quantitative Sensory Testing Responses in Patients with Chronic Nonspecific Low Back Pain? A Randomized Controlled Clinical Trial. Evid Based Complement Alternat Med 2018;2018:8586746.

18. Grover CA, McKernan MP, Close RJH. Transcutaneous Electrical Nerve Stimulation (TENS) in the Emergency Department for Pain Relief: A Preliminary Study of Feasibility and Efficacy. West J Emerg Med 2018;19(5):872-6.

19. Mendes FV, Medeiros S, Prado VFL, et al. Programa de tratamento para dor lombar crônica: uma série de casos. Movimenta 2019;12(2):262-8.

20. Dohnert MB, Borges CS, Evaldt AS, et al. Lumbopelvic Stabilization Exercises and McKenzie Method in Low Back Pain Due to Disc Protrusion: A Blind Randomized Clinical Trial. Muscles Ligaments Tendons J 2020;10(4):740-51.

21. Choopani R, Ghaderi F, Salahzadeh Z, et al. The Effect of Segmental Stabilization Exercises on Pain, Disability and Static Postural Stability in Patients with Spondylolisthesis: A Double Blinded Pilot Randomized Controlled Trial. Muscles Ligaments Tendons J 2019;9(4):615-26.
22. Awad MA, Allah AHAA. Effect of Electroacupuncture Versus Low Level Laser Therapy on Postnatal Low Back Pain. Med J Cairo Univ 2018;86(7):4125-35.

23. Kong J, Puetz C, Tian L, et al. Effect of Electroacupuncture vs Sham Treatment on Change in Pain Severity Among Adults With Chronic Low Back Pain A Randomized Clinical Trial. JAMA 2020;3(10):e2022787.

24. Zhang RY, Zhu BF, Wang LK, et al. Electroacupuncture alleviates inflammatory pain via adenosine suppression and its mediated substance P expression. Arq Neuro-Psiquiatr 2020;78(10):617-23.

25. Heo I, Hwang MS, Hwang EH, et al. Electroacupuncture as a complement to usual care for patients with non-acute low back pain after back surgery: a pilot randomised controlled trial. BMJ Open 2018;8(5):e018464.

26. Dohnert MB, Borges CS, Evaldt AS, et al. Neuromuscular electrical stimulation associated with core stability exercises in nonspecific postural low back pain: a randomized clinical trial. Muscles Ligaments Tendons J 2020;10(4):740-51.

27. Song WS, Seo H, Shin H. Effects of Electric Stimulation with Static Stretching on Hamstrings Flexibility. J Kor Phys Ther 2015;27(3):164-8

28. Armando L, Vidal BC, Renovato FJ, et al. Comparison Between Transcutaneous Electrical Nerve Stimulation and Stabilization Exercises in Fatigue and Transversus Abdominis Activation in Patients With Lumbar Disk Herniation: A Randomized Study. J Manipulative Physiol Ther 2018;41(4):323-31.

29. Tang S, Qian X, Zhang Y, Liu Y. Treating low back pain resulted from lumbar degenerative instability using Chinese Tuina combined with core stability exercises: A randomized controlled trial. Complement Ther Med 2016;25:45-50.

30. Laybidy SI, Shahmahmoodi T, Rahimi A. Therapeutic Electrical Stimulation Currents in Chronic on-specific Low Back Pain: Designing a Systematic Review. Muscles Ligaments Tendons J 2020;10(3):493-8. 